\title{
Impact of Temperature on Structural Changes in nsp2 and nsp3 of CHIKV: Molecular Dynamics Simulations
}

\author{
Durgesh Kumar \\ University of Delhi \\ Mahendra Kumar Meena \\ University of Delhi \\ Vinod Kumar \\ Jawaharlal Nehru University \\ Prashant Singh \\ University of Delhi \\ Indra Bahadur ( $\nabla$ bahadur.indra@nwu.ac.za ) \\ North-West University
}

\section{Research Article}

Keywords: nsp2 and nsp3 of CHIKV, MD simulations, effect of temperature

Posted Date: January 17th, 2022

DOI: https://doi.org/10.21203/rs.3.rs-1252778/v1

License: (c) (1) This work is licensed under a Creative Commons Attribution 4.0 International License. Read Full License 


\section{Abstract}

Authors have considered non-structural protease-2 (nsp2) and non-structural protease-3 (nsp3) of CHIKV for the investigation as they are involved in the replication of the virus and increases the infections in the human. CHIKV protease (nsp2), has a catalytic dyad (Cys ${ }^{1013} / \mathrm{His}^{1083}$ ) that processes viral polyproteins. Further, it has also been suggested that the catalytic dyad is interchangeable to a serine (proximal) and hence making called non-papain-like cysteine protease. Similarly, nsp3 plays important role during replication of CHIKV genome. In this work, we have investigated the effect of temperature on the structural stability of nsp2 and nsp3 of CHIKV. Although, we did not get any regular patter for change in the RMSD and RMSF for the nsp3 of CHIKV and observed that RMSD for nsp3 of CHIKV is acceptable at all the temperature $(300,325$ and $350 \mathrm{~K})$ but the minimum RMSD values are obtained at $325 \mathrm{~K}$. Similarly, no regular pattern is observed for RMSF trajectories of nsp3 of CHIKV at different temperature and minimum fluctuations in the position of amino-acids at $325 \mathrm{~K}$. Further, the RMSD and RMSF trajectories for nsp2 of CHIKV are investigated and observed a regular pattern in the RMSD and RMSF trajectories generated from MD simulations on increasing the temperature. In this, RMSD values are minimum at 300 $\mathrm{K}$ while the fluctuation in the position of amino-acids are maximum.

\section{Introduction}

Chikungunya virus (CHIKV) is a mosquito-borne arboviral disease. CHIKV belongs to Togaviridae family and Alphavirus genus, consisting of enveloped, positive single-stranded RNA viruses. In humans, there is a rapid onset of CHIKV infection and typically cleared in 5-7 days. It occurs via bite of an infected Aedes aegypi/albopictus, replicates in skin and from there it disseminates to liver and joints via blood. A rapid onset of disease with no pro-dermal phase, following an incubation period of 2-4 days.[1-3] CHIKV enters into the target cells via endocytosis. Upon endocytosis, endosomes undergo conformational change in acidic environments in viral envelope and exposes E1 peptide, mediating virus-host cell membrane fusion. This allows release of viral genome. The viral mRNA translates precursor non-structural proteins (nsps) and undergo cleavage to generate nsp1-nsp4. These nsps such as nsp1 (negative strand viral RNA synthesis), nsp2 (RNA helicase), nsp3 (replicase unit), and nsp4 (viral RNA polymerase).[4-7] Nsps form the replication complex and form RNA intermediates to synthesize sub-genomic (26S) and genomic (49S) RNAs. Following this, the capsid and glycoproteins are generated, leading to viral assembly by recruiting membrane-associated glycoproteins. It consists of capsid (C) protein that forms nucleocapsid. The envelope of viral particle consists of E1 and E2 transmembrane glycoproteins that arrange into 80 spikes with three E1/2 heterodimer. The E1 and E2 facilitates membrane fusion and binding to the host cell, respectively. Its genome consists of two ORFs that are flanked by $5^{\prime}$ and $3^{\prime}$ UTRs and separated by noncoding intergenic region. The ORF1 and ORF2 encodes for nsp1-4 and structural proteins such as capsid, glycoprotein, virion channel, etc., respectively.[4, 8-11] CHIKV protease (nsp2), has a catalytic dyad (Cys $^{1013} / \mathrm{His}^{1083}$ ) that processes viral polyproteins. Further, it has also been suggested that the catalytic dyad is interchangeable to a serine (proximal) and hence making called non-papain-like cysteine protease. Similarly, nsp3 plays important role during replication of CHIKV genome.[2, 12, 13] 
Molecular dynamics (MD) simulations is a time-saving, computational approach to perform molecular docking and provides the energy for complex formation between the receptor and a small molecule. Temperature-dependent MD simulation can be performed to understand the impact of temperature on the activity of non-structural protease of CHIKV. Upon screening of a small molecule against the nsp of $\mathrm{CHIKV}$, at fluctuating temperatures, gives the binding energy for the complex formation that may vary with temperature. The temperature-dependent MD simulations would give different trajectories that can be analyzed to understand the change in the nsp. $[9,11,14-17]$ MD simulation is a computer simulationbased approach that analyze physical movements of atoms, molecules and biomacromolecules such as protein, DNA and more. It's an important and effective approach to study physical and chemical changes of small molecules. MD simulations combining with Newton's second law of motion to obtain an output of MD simulation trajectories, which are snapshots of simulated molecular system, representing atomic coordinates at specific time periods. The trajectories are generated by Newton's second law of motion for time evaluation of $n$ interacting particles.[9, 14, 18-20]

We all witness to various viral infection; have threatened the humanity. Researchers are working on the inhibition of the virus through experimental work as well as computational methodologies to get the effective or promising inhibitors. Authors have considered non-structural protease-2 (nsp2) and nonstructural protease-3 (nsp3) of CHIKV for the investigation as they are involved in the replication of the virus and increases the infections in the human. In this work, the impact of temperature on non-structural protease-2 and non-structural protease-3 of CHIKV has been investigated using the molecular dynamics simulations for $100 \mathrm{~ns}$ at 300,325 and $350 \mathrm{~K}$.

\section{Computational Calculations}

\subsection{Preparation of nsp2 and nsp3}

Crystal structure for the nsp2 and nsp3 of CHIKV has been taken from the RCSB. The PDB ID for the nsp3 and nsp2 of CHIKV are 3GPO and 3TRK with resolution of 2.397 and $1.9 \AA$. These proteases are prepared before running the molecular dynamics simulations at different temperature using the pymol and UCSF1.13.1 software. The software is used to remove the present ligands, cofactors and solvent molecules. Further, add the missing atoms as well as the incomplete chain and assign charges to the atoms in the pdb files as mentioned above. [6, 10, 20-23].

\subsection{Molecular dynamics (MD) simulations}

MD simulations of nsp2 of np3 of CHIKV are performed using AMBER 18 suite. All the atoms present in the nsp2 and nsp3 are prepared using generalized Amber Force Field (GAFF) and then the water molecules were modelled on applying the TIP3P, a force field. The details of the methodology can be found in our previous research work on the various non-structural protease of CHIKV.[9, 10, 20, 23, 24] MD simulations of nsp2 and nsp3 of CHIKV are performed for $100 \mathrm{~ns}$ at 300,325 and $350 \mathrm{~K}$. To understand the change in the structural stability and flexibility, two trajectories, named root mean square deviation (RMSD) and root mean square fluctuation (RMSF) are generated. 


\section{Result And Discussion}

RMSD is used to explain the motion of the atoms of the protease with time in nanoseconds. It provided the information for the stability on changing time at a particular temperature in terms of deviation of the position of the atoms. RMSD is a quantitative scale to investigate the similarity of dissimilarity of the protease on change the temperature. It may explain the folding or unfolding of the protease with change in temperature. Higher values of the RMSD and the RMSF may indicate the flexibility or the fluctuation of the protease on changing the temperature. RMSD and RMSF are used to differentiate the flexible from the rigid structure of the nsp of CHIKV.

\subsection{RMSD analysis of nsp2 and nsp3 of CHIKV}

In this work, the divergence is not seen from the beginning and occurs after some time and shows the equilibration is done satisfactorily. MD simulations of nsp3 of CHIKV is performed at 300,325 and $350 \mathrm{~K}$ as in Figure 1. From the RMSD trajectories, it can be easily seen that RMSD for nsp3 of CHIKV value at all temperature is acceptable. Actually, no regular pattern has been observed for the nsp3 of CHIKV on varying the temperature as the RMSD values are higher at 300 and $350 \mathrm{~K}$ than at the $325 \mathrm{~K}$. It indicates that at $325 \mathrm{~K}$, there is less deviation means the structure of the nsp3 of CHIKV is compact; while the deviation is maximum at $300 \mathrm{~K}$ and has less compact structure. This information suggest that stability of the protease is maximum at $325 \mathrm{~K}$.

Then, RMSD trajectories are generated from MD simulations of nsp2 of CHIKV as in Figure 2. We have obtained a patter for the values of RMSD of nsp2 of CHIKV on increasing the temperature. The interesting part if on increasing the temperature, the RMSD values for the nsp3 of CHIKV increases and at $350 \mathrm{~K}$, the RMSD values increases a lot and crossed $3 \AA$ and not acceptable. Although, the value of deviation at 325 are acceptable but still are much higher than at $300 \mathrm{~K}$. So, it can be concluded that nsp2 of CHIKV at 300 $\mathrm{K}$ has a compact structure and stable.

\subsection{RMSF analysis of nsp2 and nsp3 of CHIKV}

Based on satisfactory equilibration, the structure of the protease fluctuated from the stable conformation of the protease of interest. This is also used to understand the stability of the protease based on the fluctuation in the amino acids. Based on the Figure 3 , it can be understood that there is no regular patter in the fluctuation of amino-acids on changing the temperature. The minimum fluctuations in the aminoacids of nsp3 of CHIKV is observed at $325 \mathrm{~K}$. It corroborates with information obtained from the RMSD trajectories of nsp3 of CHIKV at different temperature (300, 325 and $350 \mathrm{~K})$.

Now, trajectories of RMSF for nsp2 of CHIKV are generated from the MD simulations as in Figure 4. A regular pattern is observed in the fluctuation of amino-acids of nsp2 of CHIKV on increasing the temperature. Herein, the maximum fluctuations are observed at $300 \mathrm{~K}$ for the residues $60-70,90-120$, $140200,230-265$ and 270-290. With increase in temperature, the fluctuation in the amino-acids decreases. 


\section{Conclusion}

In the present work, we have investigated the effect of temperature on the structural stability of nsp2 and nsp3 of CHIKV. We did not get any regular patter for change in the RMSD and RMSF for the nsp3 of CHIKV and observed that RMSD for nsp3 of CHIKV is acceptable at all the temperature $(300,325$ and 350 $\mathrm{K}$ ) but the minimum RMSD values are obtained at $325 \mathrm{~K}$. Similarly, no regular pattern is observed for RMSF trajectories of nsp3 of CHIKV at different temperature and minimum fluctuations in the position of amino-acids at $325 \mathrm{~K}$. Further, the RMSD and RMSF trajectories for nsp2 of CHIKV are investigated and observed a regular pattern in the RMSD and RMSF trajectories generated from MD simulations on increasing the temperature. In this, RMSD values are minimum at $300 \mathrm{~K}$ while the fluctuation in the position of amino-acids are maximum.

\section{Declarations}

Author Contributions: All authors have read and approved the version of the manuscript. Experiments are performed by [Durgesh Kumar] and [Mahendra Kumar Meena]. The manuscript was written by [Vinod Kumar] and [Prashant Singh].

Funding There is no source of financial support to perform the work.

Institutional Review Board Statement: Not applicable

Disclosure of potential conflicts of interest and informed consent: I further confirm that the order of authors listed in the manuscript has been approved by all of us. The authors have relevant financial and non-financial interests to disclose.

Research involving Human Participants and/or Animals It is declared that no human participants and/or animals are used in this work.

Acknowledgement Durgesh Kumar is thankful to Professor B. Jayaram, Incharge, SCFBio, Indian Institute of Technology, Delhi for the access of the facilities and training.

\section{References}

1. Ghildiyal R, Gabrani R (2020) Antiviral therapeutics for chikungunya virus. Expert Opin. Ther. Pat. 30

2. Kumar D, Kumari K, Chandra R, et al (2021) A review targeting the infection by CHIKV using computational and experimental approaches. J. Biomol. Struct. Dyn.

3. Rodrigues AM, Souza RRM, Fonseca LMDS, et al (2020) Genomic surveillance of the chikungunya virus (CHIKV) in Northeast Brazil after the first outbreak in 2014. Rev Soc Bras Med Trop 53:. https://doi.org/10.1590/0037-8682-0583-2019

4. Chan $\mathrm{Y}, \mathrm{Teo} T$, Utt A, et al (2019) Mutating chikungunya virus non-structural protein produces potent live-attenuated vaccine candidate. EMBO Mol Med 11:. https://doi.org/10.15252/emmm.201810092 
5. Kumar S, Kumar A, Mamidi P, et al (2018) Chikungunya nsP1 directly interacts with nsP2 and modulates its ATPase activity. VirusDisease 29:

6. Kumar D, Meena MK, Kumari K, et al (2021) Exploring the effect of temperature on inhibition of nonstructural protease 3 of Chikungunya virus using molecular dynamics simulations and thermodynamics parameters. J Mol Liq 335:. https://doi.org/10.1016/j.molliq.2021.116164

7. Vishvakarma VK, Chandra R, Singh $P$ (2020) An experimental and theoretical approach to understand Fever, DENF \& its cure. Infect Disord - Drug Targets 20:. https://doi.org/10.2174/1871526520999200905122052

8. Lani R, Hassandarvish P, Chiam CW, et al (2015) Antiviral activity of silymarin against chikungunya virus. Sci Rep 5:. https://doi.org/10.1038/srep11421

9. Kumar D, Kumari K, Jayaraj A, Singh P (2020) Development of a theoretical model for the inhibition of nsP3 protease of Chikungunya virus using pyranooxazoles. J Biomol Struct Dyn 38: https://doi.org/10.1080/07391102.2019.1650830

10. Kumar D, Singh P, Jayaraj A, et al (2019) A Theoretical Model to Study the Interaction of ErythroNoscapines with nsP3 protease of Chikungunya Virus. ChemistrySelect 4:. https://doi.org/10.1002/slct.201803360

11. Singh P, Kumar D, Vishvakarma VK, et al (2019) Computational approach to study the synthesis of noscapine and potential of stereoisomers against nsP3 protease of CHIKV. Heliyon 5:. https://doi.org/10.1016/j.heliyon.2019.e02795

12. Cunha MS, Costa PAG, Correa IA, et al (2020) Chikungunya Virus: An Emergent Arbovirus to the South American Continent and a Continuous Threat to the World. Front. Microbiol. 11

13. Das PK, Puusepp L, Varghese FS, et al (2016) Design and validation of novel chikungunya virus protease inhibitors. Antimicrob Agents Chemother 60:. https://doi.org/10.1128/AAC.01421-16

14. Kumar D, Kumari K, Jayaraj A, et al (2021) Understanding the binding affinity of noscapines with protease of SARS-CoV-2 for COVID-19 using MD simulations at different temperatures. J Biomol Struct Dyn 39:. https://doi.org/10.1080/07391102.2020.1752310

15. Chandra A, Chaudhary M, Qamar I, et al (2021) In silico identification and validation of natural antiviral compounds as potential inhibitors of SARS-CoV-2 methyltransferase. J Biomol Struct Dyn. https://doi.org/10.1080/07391102.2021.1886174

16. Sharma S, Deep S (2020) In-silico drug repurposing for targeting SARS-CoV-2 main protease (Mpro). J Biomol Struct Dyn. https://doi.org/10.1080/07391102.2020.1844058

17. Vishvakarma VK, Shukla N, Reetu, et al (2019) A model to study the inhibition of nsP2B-nsP3 protease of dengue virus with imidazole, oxazole, triazole thiadiazole, and thiazolidine based scaffolds. Heliyon 5:. https://doi.org/10.1016/j.heliyon.2019.e02124

18. Maurya AK, Mishra N (2021) In silico validation of coumarin derivatives as potential inhibitors against Main Protease, NSP10/NSP16-Methyltransferase, Phosphatase and Endoribonuclease of SARS CoV-2. J Biomol Struct Dyn 39:. https://doi.org/10.1080/07391102.2020.1808075 
19. Kurt B, Temel H (2021) Development of AMBER parameters for molecular dynamics simulations of boron compounds containing aromatic structure. Chem Phys Lett 775:.

https://doi.org/10.1016/j.cplett.2021.138656

20. Kumar D, Meena MK, Kumari K, et al (2020) In-silico prediction of novel drug-target complex of nsp3 of CHIKV through molecular dynamic simulation. Heliyon 6:.

https://doi.org/10.1016/j.heliyon.2020.e04720

21. Meena MK, Kumar D, Kumari K, et al (2021) Promising inhibitors of nsp2 of CHIKV using molecular docking and temperature-dependent molecular dynamics simulations. J Biomol Struct Dyn. https://doi.org/10.1080/07391102.2021.1873863

22. Kumar D, Singh P, Jayaraj A, et al (2020) Selective Docking of Pyranooxazoles Against nsP2 of CHIKV Eluted Through Isothermally and Non-Isothermally MD simulations. ChemistrySelect 5:. https://doi.org/10.1002/slct.202000768

23. Meena MK, Kumar D, Jayaraj A, et al (2020) Designed thiazolidines: an arsenal for the inhibition of nsP3 of CHIKV using molecular docking and MD simulations. J Biomol Struct Dyn.

https://doi.org/10.1080/07391102.2020.1832918

24. Case Ross C Walker DA, Darden Junmei Wang Robert Duke TE Amber 2018 Reference Manual Principal contributors to the current codes

\section{Figures}

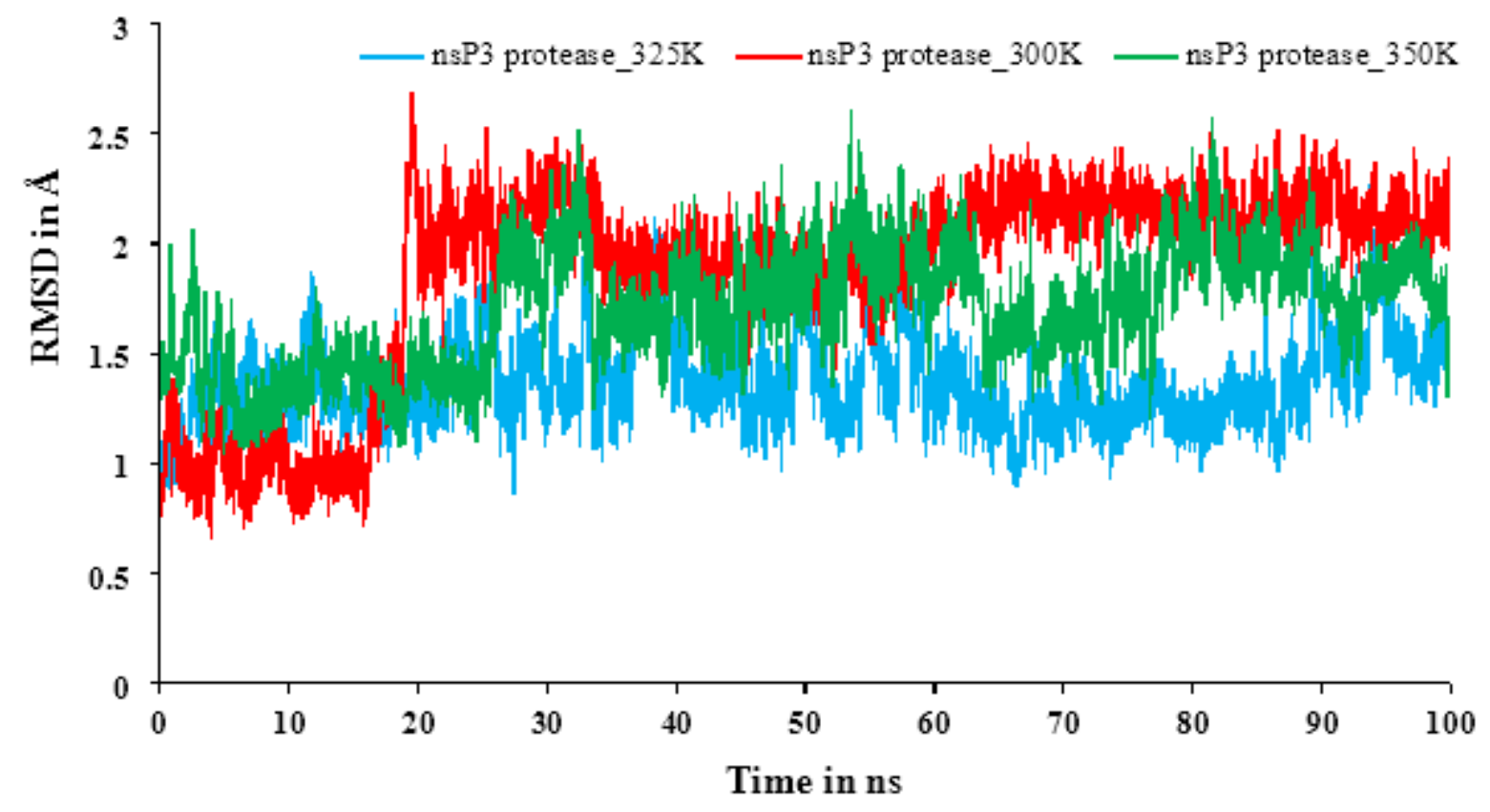

\section{Figure 1}

RMSD plot of nsp3 of CHIKV at 300, 325 and $350 \mathrm{~K}$. 


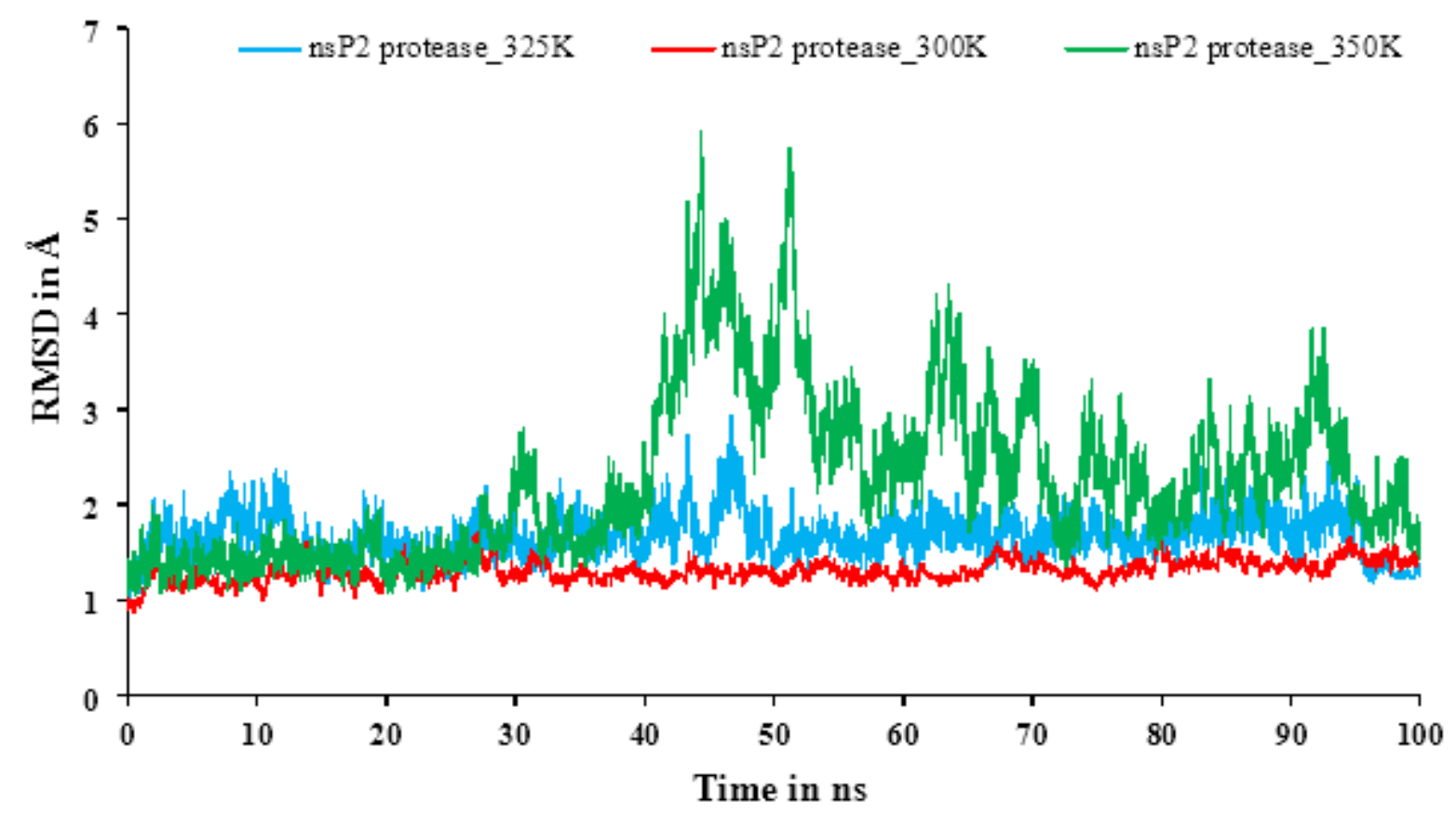

Figure 2

RMSD plot of nsp2 of CHIKV at 300,325 and $350 \mathrm{~K}$.

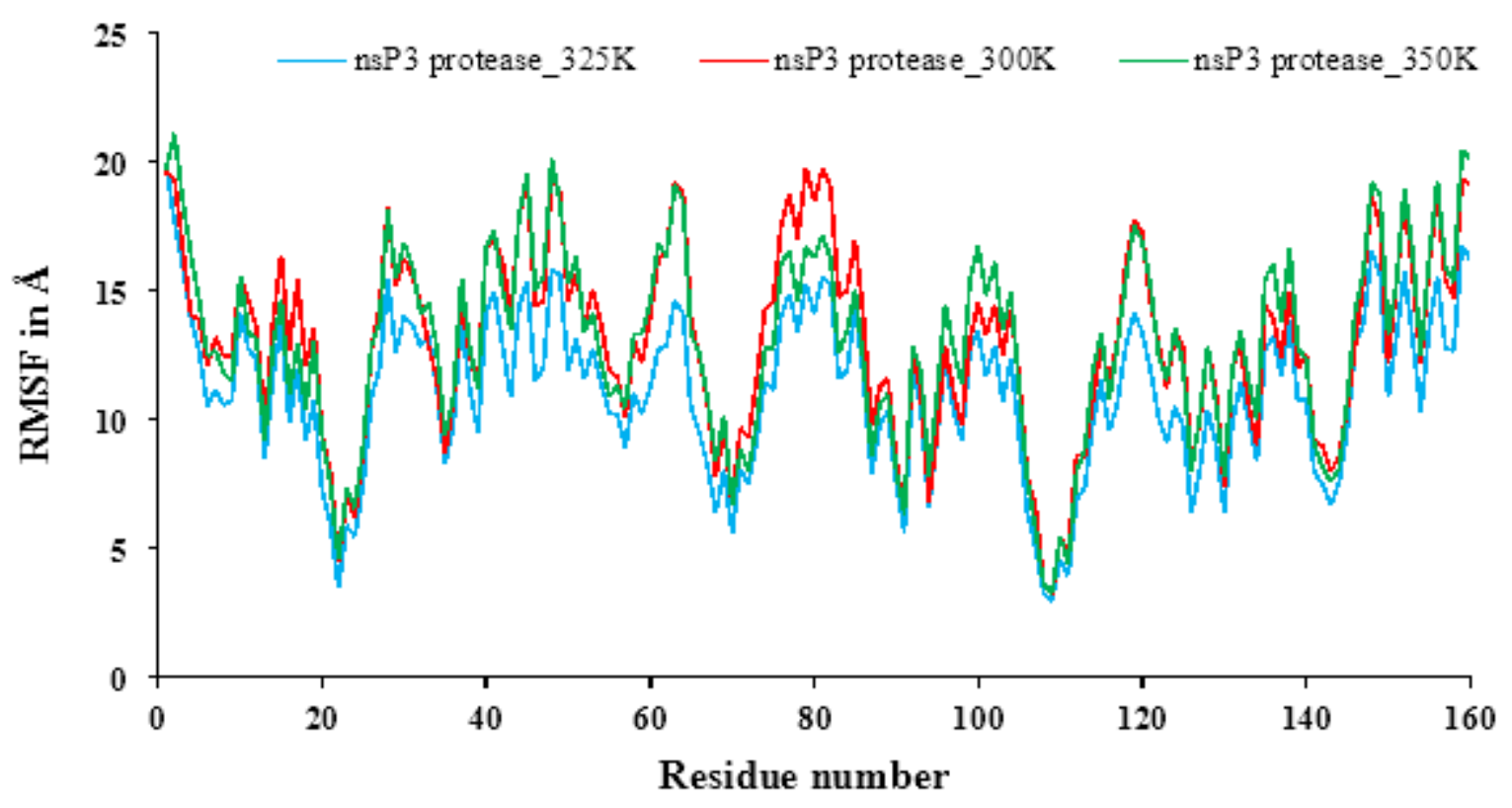

Figure 3

RMSF plot of nsp3 of CHIKV at 300,325 and $350 \mathrm{~K}$. 


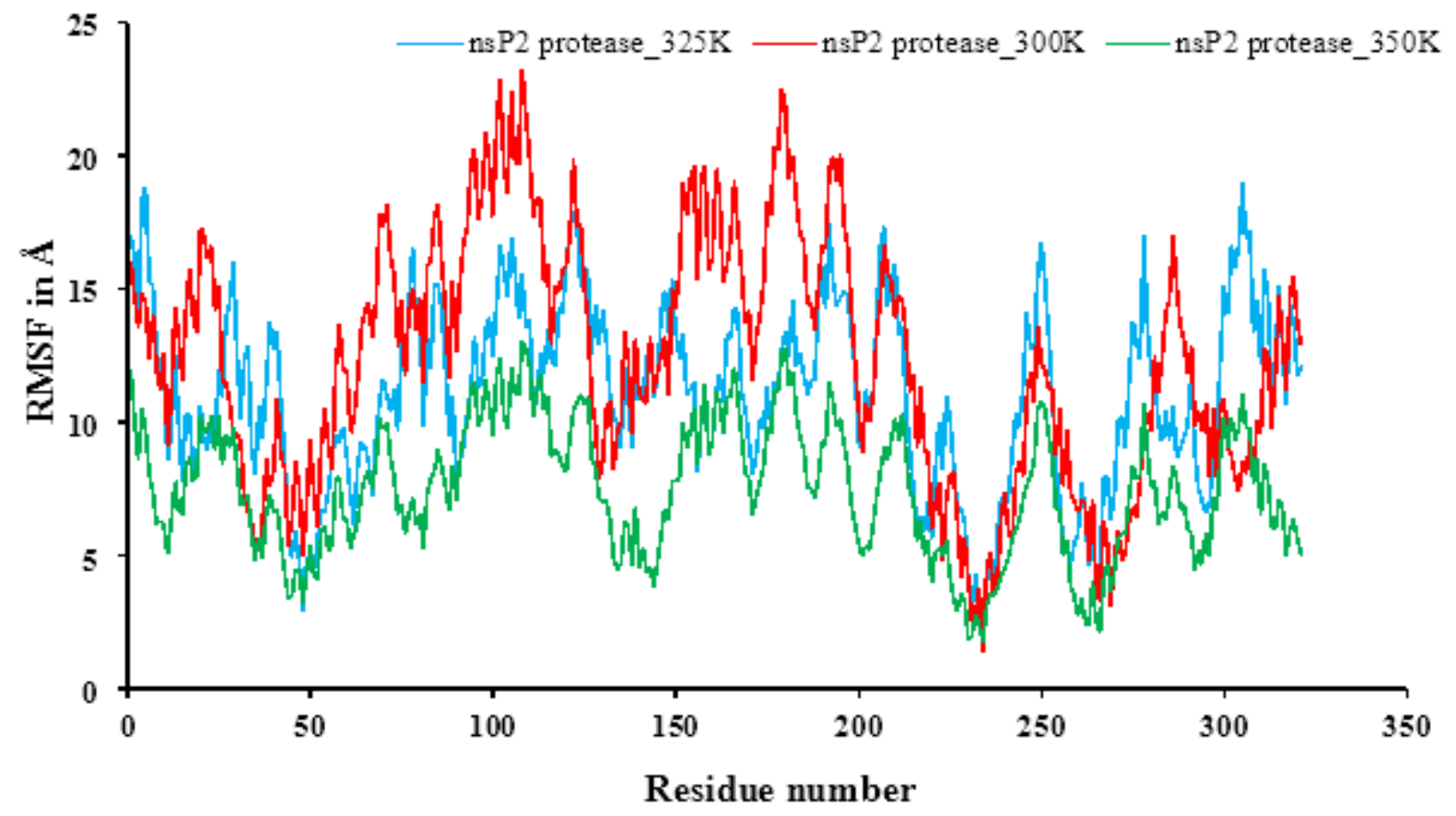

Figure 4

RMSF plot of nsp2 of CHIKV at 300, 325 and $350 \mathrm{~K}$.

\section{Supplementary Files}

This is a list of supplementary files associated with this preprint. Click to download.

- GraphicalAbstract.jpg 